\title{
Solving a Fully Fuzzy Multiobjective Programming Problem using its Equivalent Weighted Goal Programming Problem
}

\author{
Babita Mishra \\ Department of Mathematics, \\ Banaras Hindu University, Varanasi-221005
}

\author{
S. R. Singh \\ Department of Mathematics, \\ Banaras Hindu University, Varanasi-221005
}

\begin{abstract}
This paper introduces a computational method of solving fully fuzzy multi objective linear programming problem through goal programming approach. Here we deal the imprecise parameters as fuzzy numbers with assumption that these fuzzy numbers have some possibility distribution associated with fuzzy variables. In the study, we extend the concept of conflict and non-conflict between objective functions to fuzzy objective functions to compute the expected priority structure and expected aspiration level for various goals. Further, in view of some risk taken by decision maker, $\beta$ - feasibility of decision vector has been used to obtain solution for the problem. The method has been illustrated by an example and results obtained have been compared with existing solutions to show its superiority.
\end{abstract}

\section{General Terms}

Fully fuzzy multi objective linear programming problem

\section{Keywords}

Fully fuzzy multi objective linear programming problem, conflict and non-conflict between objective functions, triangular fuzzy number.

\section{INTRODUCTION}

In modelling a real life decisions making problem, one of major concern is the imprecision of information/data. As a matter of fact in many productions planning problem, one is often encountered by a situation when the information about parameters of the model are imprecise or vague. Zadeh [22] considered this problem of vagueness in information and extended the set theory to fuzzy set. Further, Zadeh introduced a method to handle such imprecision through possibility theory and considered that fuzzy parameters associated with possibility distributions may be represented as fuzzy numbers. The subject has been envisaged by several researchers and

consequent upon several methods have been developed to solve possibilistic linear programming problems by authors like Buckley [6,7], Angiz et al. [2], Akoz and Petrovic [1], Lai and Hwang [14], Arenas et.al. [3,4,5] and Jimenez et. al.[13]. Recently Chopra and Saxena [10] have given an approach to solve a possibilistic programming problem. They have considered a possibilistic linear programming involving multiple objective $s$ of conflicting nature. In approximation of fuzzy numbers by its crisp values, Heilpern [12] considered the expected values of fuzzy numbers and the concept was used by many researchers in optimization problems containing fuzzy numbers as parameters. In solving the multi objective programming with fuzzy parameters, fuzzy goal programming method appeared as one of the powerful method and have been applied by several researchers like Chen and Tsai [9], Lin[16], Yaghoobi and Tamiz [21]. Lu et al. [17] gave a concept of $\alpha$ - fuzzy goal approximate algorithm for solving fuzzy multi objective linear programming problem. Recently, Cheng et al [8] solved fuzzy multi objective linear programming problem using deviation degree measures and weighted max-min method.

Here we shall consider a Multi objective linear programming problem (MOLPP) in which all parameters are imprecise and these parameters are being represented by fuzzy numbers described by their possibility distribution. In the subsequent section of the paper, we shall give some definitions, theorems and proposition for its solution. We generalize Cohon [11] and Mohanty et. al. [20] concept of conflict between objectives to fuzzy objectives functions to find expected aspiration level of each of fuzzy objectives and their expected weights. Cocept of conflict and non conflict of objectives has also been considered by Mishra \& singh [18] and Mishra et.al. [19] for finding the appropriate aspiration level and weight of the objectives. Further, we used the $\beta$ - feasibility of Arenas et al. $[3,4]$ to obtain an equivalent weighted goal programming problem to find the solution of the fully fuzzy multi objective linear programming problem (MOLPP). The developed method has been implemented on an example for illustration

\section{FULLY FUZZY MULTI OBJECTIVE LINEAR PROGRAMMING PROBLEM}

2.1 A fully fuzzy multi objective programming problem having all parameters as fuzzy numbers can be defined as

$$
\begin{array}{ccc}
\max / \min & \tilde{\mathrm{Z}}=\tilde{\mathrm{C} X} & \\
\text { such that } & \widetilde{\mathrm{A} X} \approx \widetilde{\mathrm{b}_{j}} & \mathrm{j}=1,2, \ldots, \mathrm{k} . \\
& \widetilde{\mathrm{A} X} \approx \widetilde{\mathrm{b}_{j}} & \mathrm{j}=\mathrm{k}+1, \ldots, \mathrm{p} . \\
& \widetilde{\mathrm{A} X} \approx \widetilde{\mathrm{b}_{j}} & \mathrm{j}=\mathrm{p}+1, \ldots, \mathrm{m} .
\end{array}
$$

where $\quad \tilde{\mathrm{Z}}=\left(\widetilde{\mathrm{Z}_{1}}, \widetilde{\mathrm{Z}_{2}}, \ldots, \widetilde{\mathrm{Z}_{\mathrm{n}}}\right)$ and $\tilde{\mathrm{C}}=\left(\widetilde{\mathrm{C}_{1}}, \widetilde{\mathrm{C}_{2}}, \ldots, \widetilde{\mathrm{C}_{\mathrm{n}}}\right)^{\mathrm{t}}$

and $\mathrm{X}$ is a decision vector with components $\mathrm{X}=\left(\mathrm{x}_{1}, \mathrm{x}_{2}, \ldots, \mathrm{x}_{\mathrm{k}}\right)$. Here, imprecise parameters $\widetilde{a_{\mathrm{kj}}}$ of technological matrix $\widetilde{A}$ and $b_{j}$ are represented by fuzzy numbers being described by their possibility distributions as $\widetilde{\mathrm{a}_{\mathrm{kj}}}=\left(\mathrm{a}_{\mathrm{kj}}^{\mathrm{l}}, \mathrm{a}_{\mathrm{kj}}, \mathrm{a}_{\mathrm{kj}}^{\mathrm{u}}\right)$, where $\widetilde{\mathrm{a}_{\mathrm{kj}}}$ are the components of vector $\widetilde{A}$ and $\widetilde{b_{j}}=\left(b_{j}^{l}, b_{j}, b_{j}^{u}\right)$, Such that $a_{k j}$ and $b_{j}$ are most possible values (central values) and $a_{k j}^{l}, a_{k j}^{u}$ and $b_{j}^{l}, b_{j}^{u}$ are possible deviated value from left and from right side of central value.

Since, a fuzzy number is a fuzzy set on the real line, which is normal and convex with bounded support, hence fuzzy 
number $\widetilde{\mathrm{N}}$, in its $\alpha$-level set $\mathrm{N}_{\alpha}$ can be represented by closed interval $\left[\mathrm{N}_{\alpha}^{\mathrm{L}}, \mathrm{N}_{\alpha}^{\mathrm{R}}\right]$.

One approach for solving problem (1) is beginning with the solution of single objective fully fuzzy linear programming problem taking one objective at a time and thus we have to solve $\mathrm{n}$ sub problems (PLPP) as follows

$$
\begin{array}{ccc}
\max / \min & \widetilde{\mathrm{Z}_{\mathrm{i}}}=\widetilde{\mathrm{C}_{\mathrm{i}} \mathrm{X}} & \mathrm{i}=1,2, \ldots, \mathrm{n} \\
\text { such that } & \widetilde{\mathrm{A}_{\mathrm{j}} \mathrm{X}} * \widetilde{\mathrm{b}_{j}} & \mathrm{j}=1,2, \ldots, \mathrm{m}
\end{array}
$$

Where $(*)$ can be defined as one of $\gtrsim$ or $\lesssim$ or $\approx$.

\subsection{Pareto Optimal Solution of Subproblems}

We can define membership functions of the fuzzy set $\tilde{\mathbf{z}}^{*}$ that permits us to define a Pereto optimal solution of the fuzzy multi objective programming problem

$\mu\left(\tilde{\mathrm{z}}^{*}=\mathrm{z}^{*}\right)=$ $\sup _{A, b, C}\left\{\mu(A, b, C) \mid z^{*}\right.$ is a pareto optimal solution of MOLP $\left.(A, b, C)\right\}$

Where MOLP (A,b,C) is crisp problem associated with the Parameters A,b,C

We will construct the membership functions of a pareto optimal solutions in terms of its $\alpha$ - cuts.

$\forall(A, b, C) \in R^{m+k} \times R^{m} \times R^{k+n} \quad$ we shall define the $\left[\mathrm{z}^{*}(\mathrm{~A}, \mathrm{~b}, \mathrm{C})\right]$ set formed by all pareto optimal solutions of the $\operatorname{MOLP}(\mathrm{A}, \mathrm{b}, \mathrm{C})$ i.e.

$\left[\mathrm{z}^{*}(\mathrm{~A}, \mathrm{~b}, \mathrm{C})\right]=$

$\left\{\mathrm{z}^{*} \in \mathrm{R}^{\mathrm{n}} \mid \mathrm{z}^{*}\right.$ is a pareto optimal solution of $\left.\operatorname{MOLP}(\mathrm{A}, \mathrm{b}, \mathrm{C})\right\}$

$\forall \alpha \in[0,1]$ we denote $\Omega(\alpha)$, the set made up of all th subsets of the POSs $\left[\mathrm{z}^{*}(\mathrm{~A}, \mathrm{~b}, \mathrm{C})\right]$ associated to all possible $(\mathrm{A}, \mathrm{b}, \mathrm{C})$ of the $\alpha$ level set i.e. $\Omega(\alpha)=\left\{\left[\mathrm{z}^{*}(\mathrm{~A}, \mathrm{~b}, \mathrm{C})\right] \mid(\mathrm{A}, \mathrm{b}, \mathrm{C}) \in(\mathrm{A}, \mathrm{b}, \mathrm{C})_{\alpha}\right\}$

Where $(\mathrm{A}, \mathrm{b}, \mathrm{C})_{\alpha}=\left[\mathrm{A}_{\alpha}^{\mathrm{L}}, \mathrm{A}_{\alpha}^{\mathrm{R}}\right] \times\left[\mathrm{b}_{\alpha}^{\mathrm{L}}, \mathrm{b}_{\alpha}^{\mathrm{R}}\right] \times\left[\mathrm{C}_{\alpha}^{\mathrm{L}}, \mathrm{C}_{\alpha}^{\mathrm{R}}\right]$

is the generalized $\alpha$-cut associated to the parameters of the problem.

\subsubsection{Definition}

Let $\left[z^{*}(A, b, C)\right], z^{*}\left(A^{\prime}, b^{\prime}, C^{\prime}\right) \in \Omega(\alpha)$, We say that $\left[z^{*}(A, b, C)\right]$ is less than equal to $z^{*}\left(A^{\prime}, b^{\prime}, C^{\prime}\right)$ if $\forall z^{*} \in$ $\left[\mathrm{z}^{*}(\mathrm{~A}, \mathrm{~b}, \mathrm{C})\right] \exists \mathrm{z}^{\prime *} \in\left[\mathrm{z}^{*}\left(\mathrm{~A}^{\prime}, \mathrm{b}^{\prime}, \mathrm{C}^{\prime}\right)\right]$ s.t. $\mathrm{z}^{*} \leq \mathrm{z}^{\prime *}$

Theorem 2.2.1

Let

$(A, b, C),\left(A^{\prime}, b^{\prime}, C^{\prime}\right) \in(A, b, C)_{\alpha}$ s. t. $A \leq A^{\prime}, b \leq b^{\prime}$ and $C \leq C^{\prime}$ then

$$
\left[\mathrm{z}^{*}(\mathrm{~A}, \mathrm{~b}, \mathrm{C})\right] \leq\left[\mathrm{z}^{*}\left(\mathrm{~A}^{\prime}, \mathrm{b}^{\prime}, \mathrm{C}^{\prime}\right)\right] \quad \text { and } \quad \text { if }
$$$$
\left[\mathrm{z}_{\alpha}^{*}\right]^{\mathrm{L}}=\left[\mathrm{z}^{*}\left(\mathrm{~A}_{\alpha}^{\mathrm{L}}, \mathrm{b}_{\alpha}^{\mathrm{R}}, \mathrm{C}_{\alpha}^{\mathrm{L}}\right)=\mathrm{f}\left(\mathrm{A}_{\alpha}^{\mathrm{L}}, \mathrm{b}_{\alpha}^{\mathrm{R}}, \mathrm{C}_{\alpha}^{\mathrm{L}}\right)\right.
$$

And $\quad\left[z_{\alpha}^{*}\right]^{\mathrm{R}}=\left[\mathrm{z}^{*}\left(\mathrm{~A}_{\alpha}^{\mathrm{L}}, \mathrm{b}_{\alpha}^{\mathrm{R}}, \mathrm{C}_{\alpha}^{\mathrm{R}}\right)=\mathrm{f}\left(\mathrm{A}_{\alpha}^{\mathrm{L}}, \mathrm{b}_{\alpha}^{\mathrm{R}}, \mathrm{C}_{\alpha}^{\mathrm{R}}\right)\right.$, then $\forall \mathrm{z}^{*}\left(\mathrm{~A}^{\prime}, \mathrm{b}^{\prime}, \mathrm{C}^{\prime}\right) \in \Omega(\alpha)$ it is verified that

$$
\begin{aligned}
& {\left[\mathrm{z}_{\alpha}^{*}\right]^{\mathrm{L}} \leq\left[\mathrm{z}^{*}\left(\mathrm{~A}^{\prime}, \mathrm{b}^{\prime}, \mathrm{C}^{\prime}\right)\right]} \\
& {\left[\mathrm{z}^{*}\left(\mathrm{~A}^{\prime}, \mathrm{b}^{\prime}, \mathrm{C}^{\prime}\right)\right] \leq\left[\mathrm{z}_{\alpha}^{*}\right]^{\mathrm{R}}}
\end{aligned}
$$

These two stated inequality implies that $\Omega(\alpha)=$ $\left[\left[\mathrm{z}_{\alpha}^{*}\right]^{\mathrm{L}},\left[\mathrm{z}_{\alpha}^{*}\right]^{\mathrm{R}}\right]$

Theorem 2.2.2

Let $\alpha_{1}, \alpha_{2} \in[0,1]$ s.t. $\alpha_{1}<\alpha_{2}$ then

$$
\begin{aligned}
& {\left[\mathrm{z}_{\alpha_{1}}^{*}\right]^{\mathrm{L}}<\left[\mathrm{z}_{\alpha_{2}}^{*}\right]^{\mathrm{L}}} \\
& {\left[\mathrm{z}_{\alpha_{2}}^{*}\right]^{\mathrm{R}}<\left[\mathrm{z}_{\alpha_{1}}^{*}\right]^{\mathrm{R}}}
\end{aligned}
$$

By using above theorems we have constructed an increasing sequence of non fuzzy pareto optimal solution of the problem (2) as

$$
\mathrm{Z}_{01}^{*}, \ldots, \mathrm{Z}_{\alpha \mathrm{l}}^{*}, \ldots, \mathrm{Z}_{1 \mathrm{l}}^{*}, \mathrm{Z}_{1 \mathrm{u}}^{*}, \ldots, \mathrm{Z}_{\alpha \mathrm{u}}^{*}, \ldots, \mathrm{Z}_{0 \mathrm{u}}^{*}
$$

Here we propose the following two methods for treating equality $(\approx)$ constraints, we consider the solution of the problem with the possibility that the constraints may be of the type $\widetilde{A_{j}} X \approx \widetilde{b_{j}}$ with the following propositions.

Proposition -1 We can transform $\widetilde{\mathrm{A} X} \approx \widetilde{\mathrm{b}_{j}}$ into two inequalities as $\widetilde{\mathrm{A} X} \lesssim \widetilde{\mathrm{b}_{j}}$ and $\widetilde{\mathrm{A} X} \gtrsim \widetilde{\mathrm{b}_{j}}$, and then proceed as defined in theorem 2.2.1 and theorem 2.2.2

Proposition -2 We can convert $\widetilde{\mathrm{A} X} \approx \widetilde{\mathrm{b}_{\mathrm{j}}}$

as $A_{\alpha}=\left(A_{\alpha}^{\mathrm{L}}+\mathrm{A}_{\alpha}^{\mathrm{R}}\right) / 2$

and $b_{\alpha}=\left(b_{\alpha}^{L}+b_{\alpha}^{R}\right) / 2$

\subsection{Expected Values of Fuzzy Objectives}

The fuzzy solutions in the objective space are defined by their possibility distributions. A restriction on their possible values is considered as its expected interval and subsequently as expected values. In fact, here, the problem primarily requires values of decision variables $\mathrm{x}_{\mathrm{j}}^{*}$ to determine a fuzzy solution $\tilde{\mathrm{Z}}^{*}$. The problem is now transformed to

find $\quad x \in N\left(\widetilde{A}, \widetilde{b_{i}}\right)=\left\{x^{\in} R^{n}: \widetilde{A} x \lesssim \tilde{b}, x \gtrsim 0\right\}$

such that $\quad \widetilde{\mathrm{c}_{\mathrm{r}}} \mathrm{x}_{\mathrm{j}} \approx \tilde{\mathrm{Z}}_{\mathrm{r}}^{*}, \mathrm{r}=1, \ldots, \mathrm{k}$.

Where $\approx$ (approximately equal) is a relation between two fuzzy numbers. The expected values of $\widetilde{c_{r}}, x_{j}$ and $\tilde{Z}_{r}^{*}$ define the fuzzy goals.

Now we can compute expected values for each of the objective functions which are fuzzy numbers. The expected interval of a fuzzy number $\widetilde{\mathrm{Z}_{\mathrm{i}}}$ is defined as

$\operatorname{EI}\left(\widetilde{Z_{i}}\right)=\left[\int_{0}^{1} Z_{\mathrm{i} \alpha}^{\mathrm{L}} \mathrm{d} \alpha, \int_{0}^{1} \mathrm{Z}_{\mathrm{i} \alpha}^{\mathrm{R}} \mathrm{d} \alpha\right]$,

and expected value of a fuzzy number $\widetilde{\mathrm{Z}_{\mathrm{i}}}$ is defined as

$\operatorname{EV}\left(\widetilde{Z_{i}}\right)=\frac{\int_{0}^{1} Z_{i \alpha}^{\mathrm{L}} \mathrm{d} \alpha+\int_{0}^{1} \mathrm{Z}_{\mathrm{i} \alpha}^{\mathrm{R}} \mathrm{d} \alpha}{2}$,

\subsection{Membership Function for Expected Values of Objectives}

A pay off matrix is constructed to find max and min expected values corresponding to each objective function. Thus we can construct a membership function for expected values of objective function as

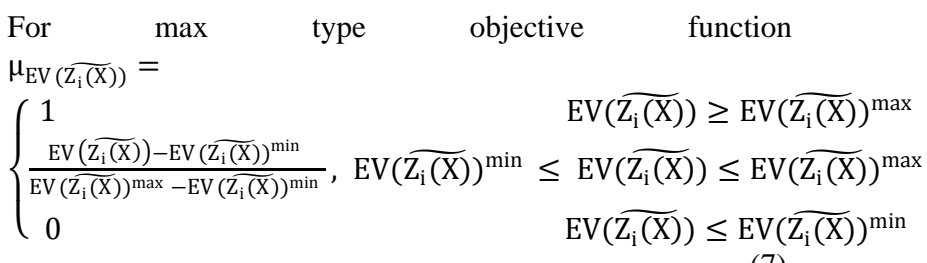

and similarly we can construct membership function for objective of min type. 


\subsection{Concept of Conflict and Non Conflict between Fuzzy Objectives}

In Multi objective programming problems, simultaneous achievement of all the objectives is not possible as they may be of conflicting in nature. The concept of conflict and non conflict given by Cohon [11] is being generalized for fuzzy objectives to compute expected priority structure and expected aspiration level to each of the objectives.

Angle between fuzzy objectives

Let $\left(\widetilde{\mathrm{C}_{\mathrm{i} 1}}, \widetilde{\mathrm{C}_{\mathrm{i} 2}}, \ldots, \widetilde{\mathrm{C}_{\mathrm{ik}}}\right)$ and $\left(\widetilde{\mathrm{C}_{\mathrm{j} 1}}, \widetilde{\mathrm{C}_{\mathrm{j} 2}}, \ldots, \widetilde{\mathrm{C}_{\mathrm{jk}}}\right)$ be the fuzzy gradients of fuzzy objectives $\widetilde{\mathrm{Z}_{i}}$ and $\widetilde{\mathrm{Z}_{j}}$ respectively, then fuzzy angle $\widetilde{\theta_{\mathrm{ij}}}$ between them can be computed as

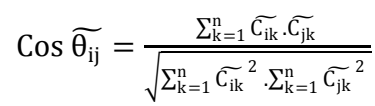

The computed, cosine angles between each of objectives are also fuzzy number and are represented as $\operatorname{Cos} \widetilde{\theta_{i j}}=(m, \alpha, \beta)$, where $m$ is modal value, $\alpha$ and $\beta$ are left and right spreads from modal value.

Thus in case of $\widetilde{\theta_{\mathrm{ij}}} \approx 0$, the simultaneous achievement of objectives $\widetilde{Z_{i}}$ and $\widetilde{Z_{j}}$ is possible but a conflict arises, when $\widetilde{\theta_{\mathrm{ij}}} \approx 0$. We can construct a membership function for non conflict between objectives $\widetilde{\mathrm{Z}_{i}}$ and $\widetilde{\mathrm{Z}_{j}}$ for their left width, right width and modal value separately.

For modal value which is

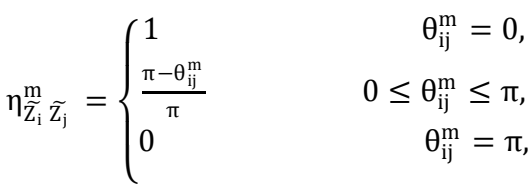

and similarly one can construct for left and right width.

\subsubsection{Non Conflict Matrix}

Since $\eta_{\widetilde{z_{i}} \widetilde{z_{j}}}$ is a fuzzy number, when $0 \lesssim \widetilde{\theta_{i j}} \lesssim \pi$, we can compute expected value for each $\eta_{\widetilde{Z_{\mathrm{i}}} \widetilde{\mathrm{Z}_{\mathrm{j}}}}$ using (5) and (6). These values can be arranged in form of a symmetric matrix named as non conflict matrix

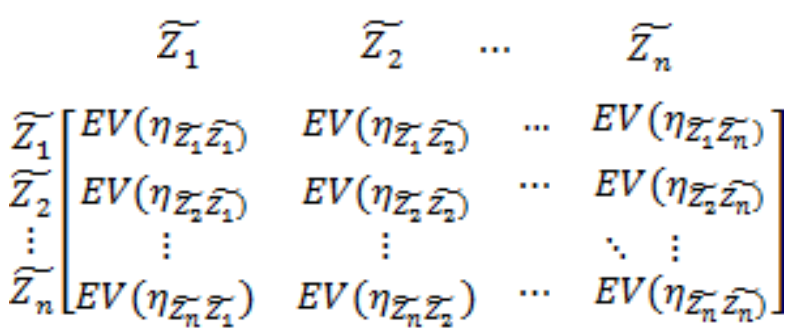

Where $\operatorname{EV}\left(\eta_{\widetilde{Z_{i}}} \widetilde{Z_{i}}\right)$ represents extent to which objective $\widetilde{Z_{i}}$ non conflicts with $\widetilde{\mathrm{Z}_{\mathrm{j}}}$ and vice versa.

\subsubsection{Appropriate priority structure}

With this matrix, we can compute expected amount of support that objective $\widetilde{\mathrm{Z}_{\mathrm{i}}}$ gets from all

other objectives as

$\operatorname{EV}\left(\widetilde{W_{i}}\right)=\frac{\sum_{j=1}^{n} E V\left(\eta_{\widetilde{z_{i}} \widetilde{z_{j}}}\right)}{n}$

Now obtained values of $\operatorname{EV}\left(\widetilde{\mathrm{W}}_{\mathrm{i}}\right)$ are crisp quantities and are ordered to give a priority structure among objectives.

\subsubsection{Appropriate aspiration level}

Further, the expected value of extent of non-conflict $\widetilde{W}_{\mathrm{i}}$ of an objective is considered as inverse of the membership function to obtain expected aspiration level and is defined as

$\operatorname{EV}\left(\widetilde{b_{i}}\right)=\left[\mu_{z_{i}^{-1}}\left(\operatorname{EV}\left(\widetilde{W_{i}}\right)\right)\right] \quad i=1,2, \ldots, n$

Now we have expected weights and expected aspiration level for each of objectives and hence can proceed to solve it by using method of weighted goal programming.

\subsection{Conversion of Fuzzy Constraints into Deterministic}

Due to fuzzy nature of elements in coefficient matrix and resource vectors, the feasibility of a decision vector can be guaranteed only by taking the intersection of all feasible set corresponding to $\alpha=0$.

i. e. $F=X \in R^{n}$, s.t. $A_{0}^{R} X \leq b_{0}^{L}, X \geq 0$

Now, if one may take some risks with respect to feasibility, with a level of tolerance in every constraint through a parameter $\beta \in[0,1]$, then such a solution obtained is considered as $\beta$ feasible solution.

\section{$2.7 \beta$ Feasibility of Decision Vector}

A decision vector $X \in F_{\beta}$, is said to be $\beta$-feasible for the problem, if $X$ verifies constraints at least in a degree $\beta$ i.e.

$\widetilde{A}_{j}(X) \leq_{\beta} \tilde{b}_{j}$, for $j=1,2, \ldots, m$ Where $\leq_{\beta}$ is defined as

$\mathrm{F}_{\beta}=$

$\left\{\begin{array}{l}{\left[A_{j}^{R}-\beta\left(A_{j}^{R}-A_{j}^{L}\right)\right] X \leq b_{j}^{L}+\beta\left(b_{j}^{R}-b_{j}^{L}\right), \quad j=1,2, \ldots, m} \\ X \geq 0,\end{array}\right.$

Where $F_{\beta}$ is the set of all $\beta$-feasible decision vectors.

\subsection{Equivalent Weighted Goal Programming}

We have calculated the appropriate expected aspiration level and expected priority structure for each of the objective function as defined in (11). Thus the problem (1) can be rewritten as

Find $\quad \mathrm{X} \in \mathrm{F}_{\beta}$

Such that $\operatorname{EV}(\tilde{\mathrm{C} X})=\operatorname{EV}(\tilde{\mathrm{b}})$

Where $\tilde{\mathrm{C}}=\left(\tilde{\mathrm{C}}_{1}, \tilde{\mathrm{C}}_{2}, \ldots, \tilde{\mathrm{C}}_{\mathrm{n}}\right)^{\mathrm{t}}$ and $\tilde{\mathrm{b}}=\left(\tilde{\mathrm{b}}_{1}, \tilde{\mathrm{b}}_{2}, \ldots, \mathrm{b}_{\mathrm{n}}\right)$

Problem (13), a deterministic weighted goal programming problem can be now solved with different values of , by any standard method.

\section{COMPUTATIONAL ALGORITHM}

Thus for solving a fully fuzzy multi objective linear programming problem by the developed method, we propose the following simplified computational algorithm:

Step 1. Convert the equality constraints into inequality constraints.

Step 2. Solve the problem as a linear programming problem taking one objective at a time with set of constraints and find the value of remaining objective functions with obtained decision variables.

Step 3. Repeat step 2 for all the objective functions, one by one. 
Step 4. Compute expected values (EV) of all the objective functions.

Step 5. Construct a pay off matrix with expected values of objective functions.

Step 6. Find maximum and minimum of expected values of each objective functions and construct membership function for each objective.

Step 7. Compute degree of non conflict between goals.

Step 8. Compute expected aspiration level for each objective function.

Step 9. Compute weight for each objective function.

Step 10. Compute grade of membership function for expected value of each objective.

Step 11. Transform the constraints into $\beta$ - feasibility form.

Step 12. Solve the equivalent goal programming problem for different values of $\beta$ to get $\beta$ - feasible solutions.

\section{NUMERICAL ILLUSTRATION}

For numerical illustration of the developed method in section 2 . and its computational algorithm in section 3 , we consider the same problem as under taken by Arenas et.al. [5] as given below:

$$
\begin{aligned}
& \max \tilde{Z}_{1}=(40,50,80) x_{1}+100 x_{2}+17 \cdot 5 x_{3} \\
& \max \tilde{Z}_{2}=(80,92,120) x_{1}+(50,75,110) x_{2}+50 x_{3} \\
& \max \tilde{Z}_{1}=(10,25,70) x_{1}+100 x_{2}+75 x_{3} \\
& \text { s.t. } \\
& 3 x_{1}+9 x_{2}+(3,8,10) x_{3} \lesssim 1000 \\
& 10 x_{1}+(7,13,15) x_{2}+15 x_{3} \lesssim 1750 \\
& (4,6,8) x_{1}+16 x_{3} \lesssim 1325 \\
& (7,12,19) x_{2}+7 x_{3} \lesssim 900
\end{aligned}
$$$$
9.5 \mathrm{x}_{1}+(3.5,9.5,11.5) \mathrm{x}_{2}+4 \mathrm{x}_{3} \approx(1060,1075,1080)
$$

Here fuzzy coefficients are characterized by triangular fuzzy numbers. We solve the above problem (14) as method proposed in section $2 \& 3$ by two approaches:

\subsection{Converting Constraints with Equality Into Inequality as Defined in Proposition-1}

By solving problem (14) as the method discussed in section $2 \& 3$,

Weight of each objective as given by (10) have been obtained as

$\operatorname{EV}\left(\widetilde{\mathrm{W}_{1}}\right)=0.8969, \mathrm{EV}\left(\widetilde{\mathrm{W}_{2}}\right)=0.8888, \mathrm{EV}\left(\widetilde{\mathrm{W}_{3}}\right)=0.8833$

and calculated expected aspiration level for each of objective function using (11) as

$\operatorname{EV}\left(\widetilde{\mathrm{Z}_{1}}(\mathrm{X})\right)=9206.39=\operatorname{EV}\left(\widetilde{\mathrm{b}_{1}}\right), \operatorname{EV}\left(\widetilde{\mathrm{b}_{2}}\right)=$ 12022.01, $\operatorname{EV}\left(\widetilde{\mathrm{b}_{3}}\right)=10776.48$

Now using (12) the set of constraints $F_{\beta}$ can be defined as

$$
\mathrm{F}_{\beta}=\left\{\begin{array}{c}
(14-8 \beta) \mathrm{x}_{1}+17 \mathrm{x}_{2} \leq 1400 \\
3 \mathrm{x}_{1}+9 \mathrm{x}_{2}+(10-7 \beta) \mathrm{x}_{3} \leq 1000 \\
10 \mathrm{x}_{1}+(15-8 \beta) \mathrm{x}_{2}+15 \mathrm{x}_{3} \leq 1750 \\
(8-4 \beta) \mathrm{x}_{1}+16 \mathrm{x}_{3} \leq 1325 \\
(19-12 \beta) \mathrm{x}_{2}+7 \mathrm{x}_{3} \leq 900 \\
9.5 \mathrm{x}_{1}+(11.5-8 \beta) \mathrm{x}_{2}+4 \mathrm{x}_{3} \leq(1060+20 \beta) \\
-9.5 \mathrm{x}_{1}-(11.5-8 \beta) \mathrm{x}_{2}-4 \mathrm{x}_{3} \leq(-1060+20 \beta)
\end{array}\right.
$$

Now the under taken problem (14) reduces to a weighted goal programming problem

as follows

find $X$

s.t.

$$
\begin{aligned}
& 55 x_{1}+100 x_{2}+17.5 x_{3}=9206.39 \\
& 96 x_{1}+77.5 x_{2}+50 x_{3}=12022.01 \\
& 32.5 x_{1}+100 x_{2}+75 x_{3}=10776.48 \\
& \text { and } X \in F_{\beta}
\end{aligned}
$$

The above weighted goal programming problem (15) has been solved by MATLAB using goal attainment method for different values of $\beta$ and the results obtained are placed in table 1

\subsection{Converting Constraints with EqualityInto Inequality as Defined in Proposition-2}

Repeating the steps already carried out in section 4.1 , we obtain solution of resultants of weighted goal programming problem for different values of $\beta$, and are placed in table- 2

\section{SUMMARY AND CONCLUSIONS}

Thus in this paper we presented a solution procedure to obtain decision vector to allow a decision maker to approximate the goals with fuzzy coefficients having various degree of risk factors. The merit of the method lies with the fact that the obtained decision vectors are crisp and incorporate the choice of decision maker. Here in order to resolve the major problem of simultaneous achievements of goals, we obtained the degree of conflict and non conflict between objectives. The method comprehensively computes weights for each objective and their respective aspiration levels. Incorporating it, the method efficiently transforms a fully fuzzy MOLPP into weighted goal programming problem which can be easily solved by any standard method.

Further, the problem of a fully fuzzy MOLPP dealing with involves the issues of feasibility and optimality. Here, the imprecise parameters are considered as fuzzy numbers having possibility distribution are handled by their expected values i.e. a crisp quantity. We obtained fuzzy ideal solution, concept of compromise programming to resolve the problem of optimality. The problem of feasibility has been acknowledged by considering comparison of fuzzy numbers using $\beta$ feasibility. Thus the results obtained by our two methods have been compared with the results of Arenas [5] as summarized in table- $3 \& 4$.

The results are placed in table $3 \& 4$ depict that the solutions by both of our methods 4.1 and 4.2 are better than that of Arenas [5]. This is due to fact that our procedure properly incorporates the conflict and non conflict between goals to provide appropriate weights and aspiration levels to each goal. 
Thus the method clearly shows its superiority over existing methods.

\section{ACKNOWLEDGEMENTS}

Authors are thankful to National Board of Higher Mathematics, Department of Atomic Energy, Govt. of India, New Delhi for providing financial support to carry out the research work.

Table-1 $\beta$-feasible solution

\begin{tabular}{|l|r|r|r|r|r|r|}
\hline$\beta$ & \multicolumn{1}{|c|}{$x_{1}$} & \multicolumn{1}{c|}{$x_{2}$} & \multicolumn{1}{c|}{$x_{3}$} & $E V\left(\widetilde{Z_{1}}\right)$ & \multicolumn{1}{c|}{$E V\left(\widetilde{Z_{2}}\right)$} & $E V\left(\widetilde{Z_{3}}\right)$ \\
\hline 0 & 56.06 & 32.41 & 38.68 & 7001.2 & 9827.535 & 7963.95 \\
\hline 0.2 & 57.35 & 35.16 & 42.76 & 7418.55 & 10368.5 & 8586.875 \\
\hline 0.4 & 73.87 & 18.44 & 50.07 & 6783.075 & 11024.12 & 8000.025 \\
\hline 0.6. & 59.49 & 47.77 & 44.11 & 8820.875 & 11618.715 & 10018.675 \\
\hline 0.8 & 72.19 & 40.45 & 39.09 & 8699.525 & 12019.615 & 9322.925 \\
\hline 1 & 92.12 & 18.99 & 25.38 & 7409.75 & 11584.245 & 6796.4 \\
\hline
\end{tabular}

Table-2 $\beta$ - feasible solution

\begin{tabular}{|l|r|r|r|r|r|c|}
\hline$\beta$ & \multicolumn{1}{|c|}{$x_{1}$} & \multicolumn{1}{c}{$x_{2}$} & \multicolumn{1}{c|}{$x_{3}$} & \multicolumn{1}{c|}{$E V\left(\widetilde{Z_{1}}\right)$} & \multicolumn{1}{c|}{$E V\left(\widetilde{Z_{2}}\right)$} & $E V\left(\widetilde{Z_{3}}\right)$ \\
\hline 0 & 56.2 & 30.61 & 43.51 & 6913.425 & 9942.975 & 8150.75 \\
\hline 0.2 & 65.12 & 25.39 & 46.62 & 6936.45 & 10550.245 & 8151.9 \\
\hline 0.4 & 61.28 & 37.78 & 42.91 & 7899.325 & 10956.33 & 8987.85 \\
\hline 0.6. & 63.58 & 45.59 & 34.96 & 8667.7 & 11384.905 & 9247.35 \\
\hline 0.8 & 99.11 & 13.22 & 9.76 & 6943.85 & 11027.11 & 5275.075 \\
\hline 1 & 95.81 & 1.66 & 40.93 & 6151.825 & 11372.91 & 6349.575 \\
\hline
\end{tabular}

Table-3 Expected values of objective functions by proposed method-I

\begin{tabular}{|c|c|c|c|c|c|c|c|c|c|}
\hline \multirow[t]{3}{*}{$\beta$} & \multicolumn{6}{|c|}{ Solution by Arenas et. al.(2005) } & \multirow{2}{*}{\multicolumn{3}{|c|}{$\begin{array}{l}\text { Solution by proposed method } \\
\text { with ( } 4.1)\end{array}$}} \\
\hline & \multicolumn{3}{|l|}{$L_{1}$} & \multicolumn{3}{|l|}{$L_{\infty}$} & & & \\
\hline & $E V\left(\widetilde{Z_{1}}\right)$ & $E V\left(\widetilde{Z_{2}}\right)$ & $E V\left(\widetilde{Z_{3}}\right)$ & $E V\left(\widetilde{Z_{1}}\right)$ & $E V\left(\widetilde{Z_{2}}\right)$ & $E V\left(\widetilde{Z_{3}}\right)$ & $E V\left(\widetilde{Z_{1}}\right)$ & $E V\left(\widetilde{Z_{2}}\right)$ & $E V\left(\widetilde{Z_{3}}\right)$ \\
\hline 0.6 & 8119.1 & 11248.6 & 9091.7 & 8266.9 & 11054.1 & 9370.4 & 8820.87 & 11618.7 & 10018.7 \\
\hline 0.8 & 6326.1 & 11206.1 & 6603.8 & 6326.1 & 11221.2 & 6438.2 & 8699.52 & 12019.6 & 9322.9 \\
\hline 1 & 6140.2 & 11175 & 6181.1 & 6140.2 & 11205.4 & 6069.9 & 7409.75 & 11584.2 & 6796.4 \\
\hline
\end{tabular}




\begin{tabular}{|c|c|c|c|c|c|c|c|c|c|}
\hline \multicolumn{10}{|c|}{ Table-4: Expected values of objective functions by proposed method-II } \\
\hline \multirow[t]{3}{*}{$\beta$} & \multicolumn{6}{|c|}{ Solution by Arenas et. al.(2005) } & \multirow{2}{*}{\multicolumn{3}{|c|}{$\begin{array}{l}\text { Solution by proposed method } \\
\text { with }(4.2)\end{array}$}} \\
\hline & \multicolumn{3}{|l|}{$L_{1}$} & \multicolumn{3}{|l|}{$L_{\infty}$} & & & \\
\hline & $E V\left(\widetilde{Z_{1}}\right)$ & $E V\left(\widetilde{Z_{2}}\right)$ & $E V\left(\widetilde{Z_{3}}\right)$ & $E V\left(\widetilde{Z_{1}}\right)$ & $E V\left(\widetilde{Z_{2}}\right)$ & $E V\left(\widetilde{Z_{3}}\right)$ & $E V\left(\widetilde{Z_{1}}\right)$ & $E V\left(\widetilde{Z_{2}}\right)$ & $E V\left(\widetilde{Z_{3}}\right)$ \\
\hline 0.6 & 8119.1 & 11248.6 & 9091.7 & 8266.9 & 11054.1 & 9370.4 & 8667.7 & 11384.9 & 9247.35 \\
\hline 0.8 & 6326.1 & 11206.1 & 6603.8 & 6326.1 & 11221.2 & 6438.2 & 6943.85 & 11027.1 & 5275.07 \\
\hline 1 & 6140.2 & 11175 & 6181.1 & 6140.2 & 11205.4 & 6069.9 & 6151.82 & 11372.9 & 6349.6 \\
\hline
\end{tabular}

\section{REFERENCES}

[1] Akoz, Onur, Petrovic, D., 2007 A fuzzy goal programming method with imprecise goal hierarchy, European Journal of Operational Research 181, 14271433.

[2] Angiz, M., Z., Saati, S., Memariani, A., Movahedi, M.,M., 2006 Solving possibilistic linear programming problem considering membership function of the coefficients, Fuzzy Sets and Systems 1(2) 131-142.

[3] Arenas, M., Bilbao, A., Rodriguez, M.,V., 1999a Solving the multiobjective possibilistic linear programming problem, European Journal of Operational Research 117, 175-182.

[4] Arenas, M., Bilbao, A., Rodriguez, M.V., 1999b Solution of a possibilistic multiobjective linear programming problem, European Journal of Operational Research 119, 338-344.

[5] Arenas, M., Bilbao, A., Gladish B.P., Rodriguez, M.V., 2005 Solving a multiobjective possibilistic problem through compromise programming, European Journal of Operational Research, 164, 748-759.

[6] Buckley, J,.J., 1988 Possibilistic linear programming with triangular fuzzy numbers. Fuzzy Sets and Systems 26, 135-138.

[7] Buckley, J.,J., 1989 Solving possibilistic linear programming problems, Fuzzy Sets and Systems 31 ,329-341.

[8] Cheng, Haifang, Huang, Weilai, Zhou, Quan, Cai Jianhu,2013 Solving fuzzy multi-objective linear programming problems using deviation degree measures and weighted max-min method, Applied Mathematical Modelling 37,6855-6869.

[9] Chen, L.,H, Tsai, F.,C, 2001 Fuzzy goal programming with different importance and priorities, European Journal of Operational Research 133, 548-556.

[10] Chopra, R, Saxena R,R , 2014 An approach to solve a possibilistic linear programming problem. Applied Mathematics 5, 226-233.

[11] Cohon, J.,L.,1978 Multiobjective programming and planning, Academic Press, New York.
[12] Heilpern, S, 1992 The expected value of a fuzzy number, Fuzzy Sets and Systems 47, 81-86.

[13] Jimenez, M., Arenas. M., Bilbao, A., Rodriguez, M.,V., 2000 Solving a possibilistic linear program through compromise programming, Mathware and Soft Computing 7(2-3), 175-184.

[14] Lai, Y.,J., Hwang, C.L., 1992 A new approach to some possibilistic linear programming problem, Fuzzy Sets and Systems 49, 121-133.

[15] Li, Shaoyuan, Hu, Chaofang, 2009 Satisfying optimization method for fuzzy multi objective optimization problem, European Journal of Operational Research 197, 675-684.

[16] Lin, C.,C., 2009 A weighted max-min model for fuzzy goal programming, Fuzzy sets and Systems 197, 675684 .

[17] Lu, Jie, Ruan, D., Wu, Fengjie, Zhang, Guangquan, 2007 An $\alpha$-fuzzy goal approximate algorithm for solving fuzzy multiple objective linear programming problems, Soft Comput, 11, 259-267.

[18] Mishra, B, Singh, S.,R., 2013 Linear fractional programming procedure for multi objective linear programming problem in agricultural system. International Journal of Computer Application 61(20), 45-52.

[19] Mishra, B., Nishad, A.,K., Singh, S.,R., 2014 Fuzzy multi objective fractional programming for landuseplanninginagriculturalproductionsystem.FuzzyIfo rmation and Engineering 6, 245-262.

[20] Mohanty, B.,K., Vijayaraghawan, T.,A.,S., 1995 A multiobjective programming problem and its equivalent goal programming problem with approprite priorities and aspiration levels:A fuzzy approach, Computers Ops. Res., 22(8), 771-778.

[21] Yaghoobi, M.,A., Tamiz, M., 2007 A method of solving fuzzy goal programming problems based on MINMAX approach, European Journal of Operational Research $177,1580-1590$.

[22] Zadeh, L.,A., 1978 Fuzzy sets as a basis of theory of possibility, Fuzzy Sets and Systems 1, 3-28. 